\title{
Zirconia Inlay on Vital Teeth with Profunda Caries
}

\author{
Sandra Kartika Sari', Kun Ismiyatin², Bagus Aji Wibowo ${ }^{3}$, Rara Amorita Miranda ${ }^{3}$ \\ ${ }^{1}$ Resident of Conservative Dentistry, Faculty of Dental Medicine, Universitas Airlangga, Surabaya, Indonesia \\ ${ }^{2}$ Department of Conservative Dentistry, Faculty of Dental Medicine, Universitas Airlangga, Surabaya, Indonesia \\ ${ }^{3}$ Undergraduate Student, Faculty of Dental Medicine, Universitas Airlangga, Surabaya, Indonesia
}

\begin{abstract}
Background: Dental caries has historically been considered the most critical component of the global burden of oral disease. Health facilities and dental health education counselling have been conducted, but public knowledge about dental caries is still low. The increasing number of dental caries is currently influenced by one of the factors of community behaviour. Most people do not realize the importance of taking care of oral and dental health. The ignorance of the community results in a decrease in productivity due to the influence of the perceived illness. Advances in dentistry since the last decade has allowed the use of conservative dental care. Modern restorative dentistry offers many methods for restoring teeth, both direct and indirect. The need for restoration of posterior teeth is related to aesthetic purposes and functional, biocompatibility and biomechanical aspects of the remaining tooth structure. Some materials that are widely used as tooth-coloured indirect restorations in posterior teeth are zirconia. Zirconia has its characteristics, especially in terms of functionality, such as mechanical strength, physical strength and aesthetics. Purpose: This study aims to determine the management of indirect restoration treatment using zirconia inlay on upper premolar. Case(s): A 46-yearold male patient complained that the filling of his upper left tooth was often loose and uncomfortable when used for eating because the food was stuck in it. The patient wants his teeth treated. The history of treatment on the tooth in question has been patched two times, but it often comes off partially. Case Management: From the examination that has been carried out, a clinical diagnosis of reversible pulpitis was established. The treatment plan that will be carried out is indirect pulp capping using MTA and resin-modified glass ionomer cement as the base material. The planned restoration treatment is a fixed inlay restoration made of monolithic zirconia. Conclusion: Recently, zirconia has also been developed staining with improved translucency so that it becomes more aesthetic. Zirconia has a higher level of material resistance than other restorative materials such as composites. This is what makes zirconia the choice, especially for use as a framework for all-ceramic and partially-fixed crowns dental prosthesis.
\end{abstract}

Keywords: indirect restoration; inlay; zirconia

Correspondence: Kun Ismiyatin, Department of Conservative Dentistry, Faculty of Dental Medicine, Universitas Airlangga. Jl. Mayjend. Prof. Dr. Moestopo no. 47, Surabaya 60132, Indonesia. E-mail: kun-is@fkg.unair.ac.id

\section{INTRODUCTION}

Dental caries is a disease that affects the hard tissues of the teeth, namely enamel, dentin and cementum, in the form of decaying areas on the teeth, resulting from gradually dissolving the mineral surface and continuing to develop into the interior of the teeth. This process occurs due to the activity of microorganisms in carbohydrates that can be fermented. This process is characterized by demineralization of hard tissue and subsequent breakdown of organic matter, allowing further bacterial invasion into the interior of the tooth, namely the dentinal layer and reaching the pulp. ${ }^{1}$ Dental caries has historically been considered the most critical component of the global burden of oral disease. Health facilities and dental health education counselling have been conducted, but public knowledge about dental caries is still low. ${ }^{2}$ The increasing number of dental caries is currently influenced by one of the factors of community behaviour. Most people do not realize the importance of taking care of oral and dental health. The ignorance of the community results in a decrease in productivity due to the influence of the perceived illness. This is due to a decrease in the supporting tissues of the teeth. Dental caries will later become a source of infection that can lead to several systemic diseases. ${ }^{3}$

Advances in dentistry since the last decade has allowed the use of conservative dental care. Modern restorative dentistry offers many methods for restoring teeth, both direct and indirect. ${ }^{4}$ Indirect restorations of posterior teeth based on shape can be divided into inlays and onlays. Inlays and onlays are indirect forms of restoration used when a molar or premolar tooth is too damaged to support the underlying 
restoration, but not so severe that it requires a crown. Inlays and onlays are prepared outside the patient's mouth, then cemented or glued to the teeth. The inlay or onlay has a shape that fits the pre-prepared tooth like a puzzle piece. Indirect restorations are intended to rebuild large areas of the tooth surface, whereas direct restorations are direct restorations designed to fill a small cavity in the tooth enamel. Inlays and onlays are not as extensive as the jacket crown, which covers most of the teeth. The inlay is placed on the occlusal surface between the cusp and the tooth, whereas the onlay covers one or more tooth cusps. ${ }^{5}$

The need for restoration of posterior teeth is related to aesthetic purposes and functional, biocompatibility and biomechanical aspects of the remaining tooth structure. Some materials that are widely used as tooth-coloured indirect restorations in posterior teeth are zirconia. Zirconia has its characteristics, especially in terms of functionality, such as mechanical strength, physical strength and aesthetics. The selection of indirect restorative materials for posterior teeth must consider the various characteristics of each material, both aesthetically and functionally, so that the suitable material can be determined. ${ }^{6}$

\section{CASE(S)}

A 46-year-old male patient complained that the filling of his upper left tooth was often loose and uncomfortable when used for eating because the food was stuck in it. The patient wants his teeth treated. The history of treatment on the tooth in question has been patched two times, but it often comes off partially. Last patched one year ago, but

Table 1. Salivary test results in patients

\begin{tabular}{lcc}
\hline Hydration & $25 \mathrm{sec}$ & $\bullet$ \\
\hline Viscosity & watery & $\bullet$ \\
\hline $\mathrm{pH}$ & 7.2 & $\bullet$ \\
\hline Quantity & $5 \mathrm{ml} / 5$ minutes & $\bullet$ \\
\hline Buffer Capacity & 10 & $\bullet$ \\
\hline
\end{tabular}

the patch only lasted about three months. There has never been a complaint of spontaneous pain, a little pain when drinking cold drinks. The patient stated that he had no systemic health disorder.

The results of intraoral examination revealed that the patient had a normal posterior cusp to fossa and anterior relation with an overjet and overbite value of $2 \mathrm{~mm}$ each. The salivary test results also showed that the quality and quantity of the patient's saliva were still within normal limits (Table 1). On objective examination, there were 24 deep carious teeth with normal gingival conditions (Figure 1). In the EPT vitality test, tooth 14 as a control reacted at number 4 , while on the vitality test, tooth 24 reacted at number 3 . It can be concluded that tooth 24 is vital. Figure 2 shows the supporting examination in radiographs showed a radiolucent image on the coronal side of tooth 24 in the distal part of the tooth without any periapical abnormalities.

From the examination that has been carried out, a clinical diagnosis of reversible pulpitis was established. The treatment plan that will be carried out is described in Figure 3, indirect pulp capping using MTA and resinmodified glass ionomer cement as the base material. The planned restoration treatment is a fixed inlay restoration made of monolithic zirconia. The treatment prognosis is good because the teeth are still vital, the remaining hard tissue is still sufficient for restoration, there are no periapical abnormalities, no systemic abnormalities, and the patient is cooperative.

\section{CASE MANAGEMENTS}

Treatment was completed in 4 visits, with each visit screening patients and antigen swabs first as a form of implementing health protocols during the pandemic. Both operators and assistant operators use level 3 personal protective equipment (PPE), and patients use protective equipment in the form of an apron and nurse cap, and the treatment is carried out in a negative pressure room equipped

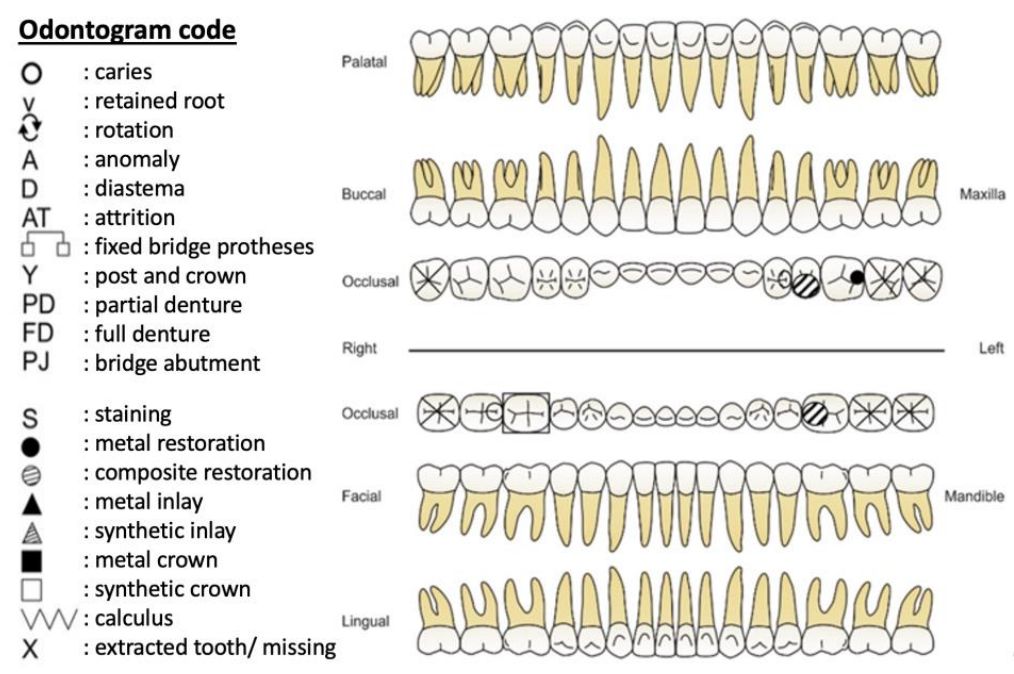

Figure 1. The results of the patient's intraoral examination are depicted through the odontogram. 
with aerosol suction (Figure 4). At visit 1 (12-7-2021), IEC and DHE were performed on the patient, and the patient received informed consent and informed consent as a form of approval for the treatment to be carried out. After the saliva test was performed, the patient was instructed to rinse his mouth with $1 \%$ povidone-iodine solution for 30 seconds, then proceed with the history and diagnosis stage. The working area was isolated using a rubber dam and dental suction, then the old composite filling was dismantled, and caries removal was performed. The next stage is indirect pulp capping using MTA material, followed by applying moist cotton pellets and temporary fillings (Figure 5).

At the second visit (26-7-2021), the patient did not feel any complaints in the anamnesis, and there were no extraoral abnormalities. The results of the intraoral examination showed that the EPT of tooth 24 reacted at number 4 the same as the EPT of tooth 14 as a control, negative percussion $(-)$, the temporary filling was still in good condition, and the gingiva around the teeth was normal. Figure 6 shows that shade taking was done using the Vita 3D Master Shade Guide 3M2. After that the work area was isolated, the temporary filling was removed, and the base was made using RMGIC.

The next stage is inlay preparation, followed by an examination of the undercut area using wax. Phosphoric acid $37 \%$ was used for etching for 10 seconds, followed by universal adhesive application on dentin and curing for 20 seconds (Figure 7). The teeth were then applied with pumice, after which the rubber dam was removed to prepare for gingival management using a retraction cord.
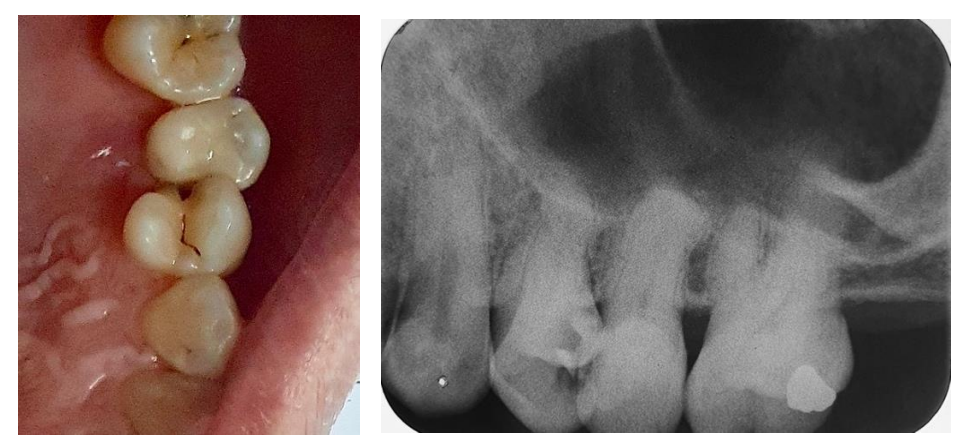

Figure 2. Initial clinical photo (left) and initial radiograph (right).
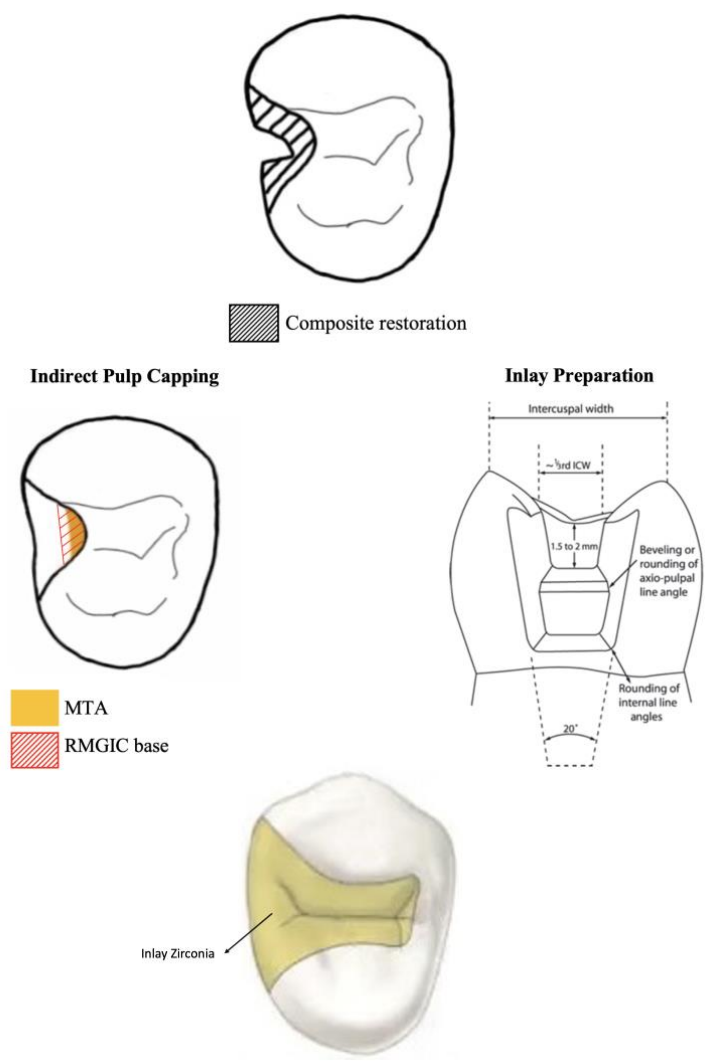

Figure 3. Schematic before treatment (top), during treatment (middle), and after treatment (bottom).

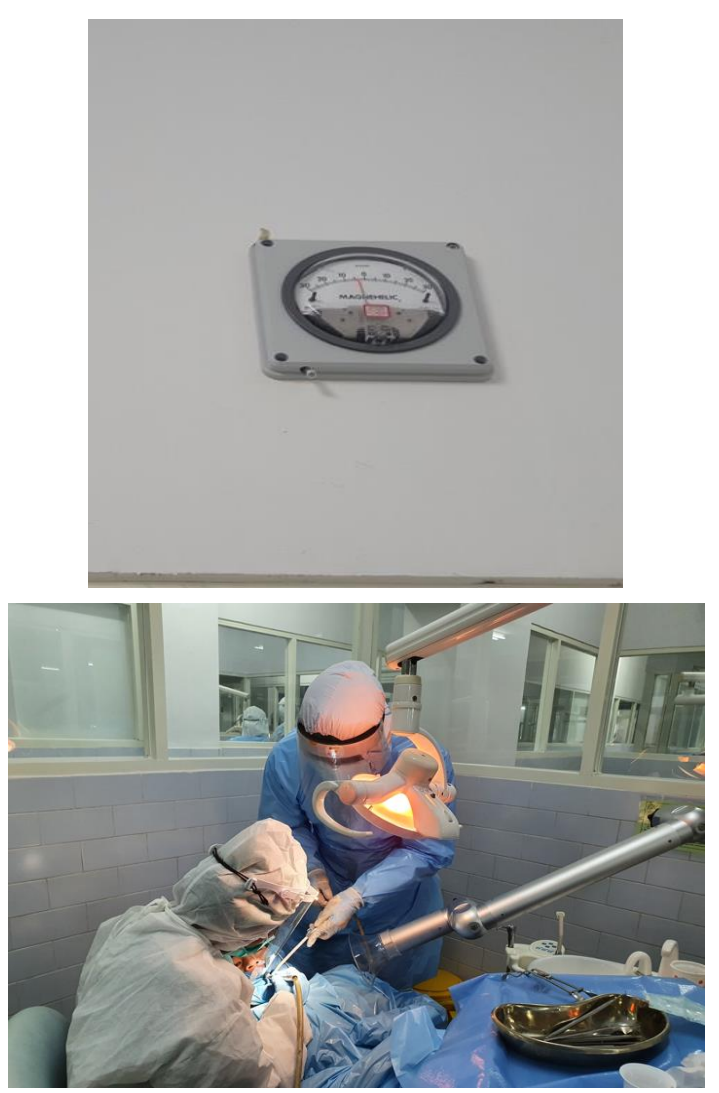

Figure 4. Treatment room (top) and documentation during treatment (bottom). 

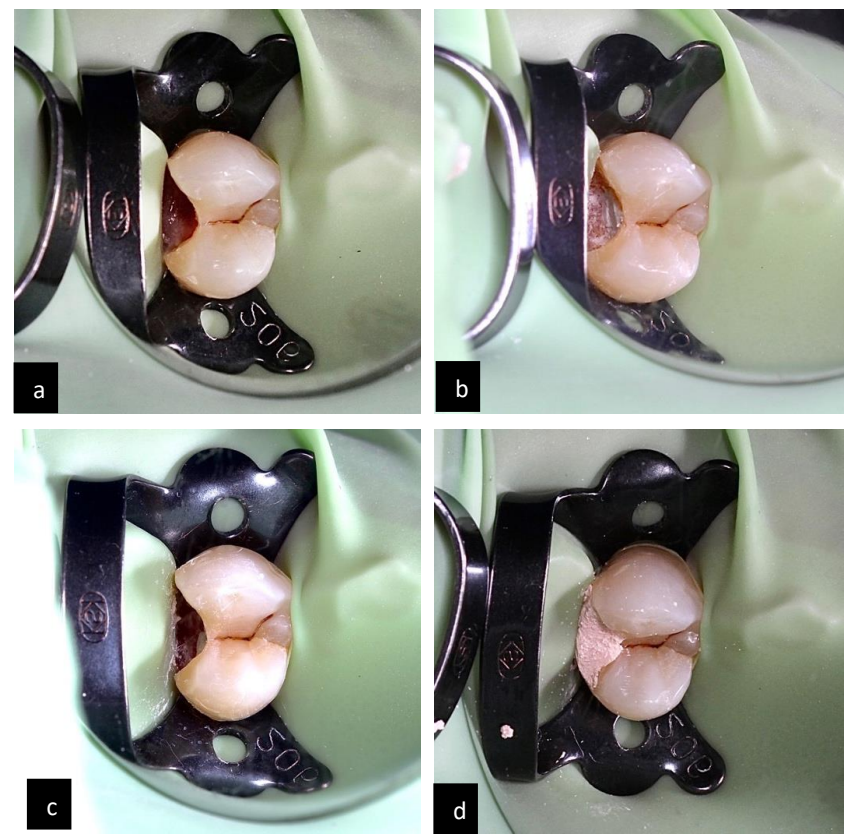

Figure 5. a) Isolation of the work area; b) Indirect pulp capping; c) Apply moist cotton pellets; d) Temporary filling.
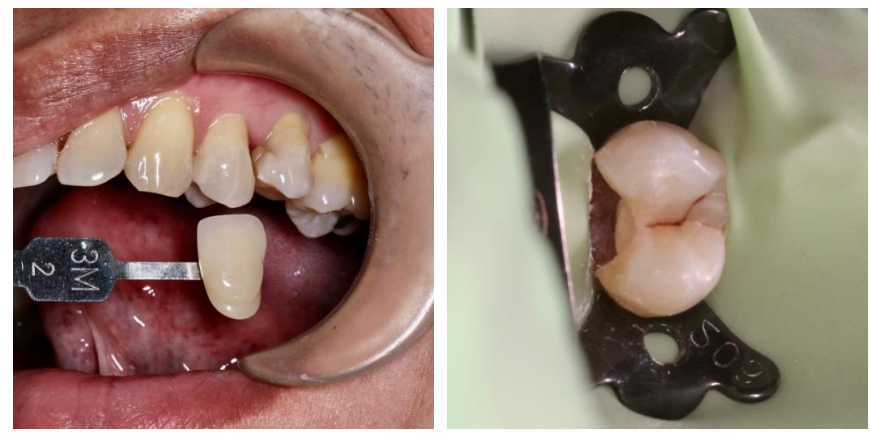

Figure 6. Shade taking (left) and base RMGIC (right).
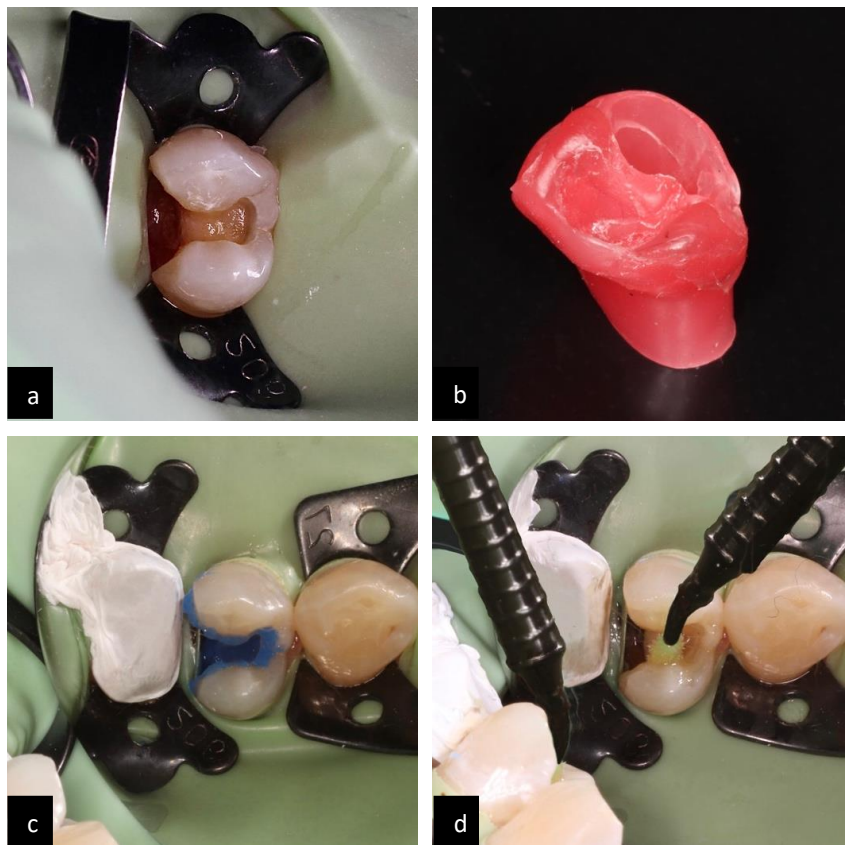

Figure 7. a) Inlay preparation; b) Checking undercuts; c) Total etch; d) Universal adhesive. 
The maxillary working model was printed using polyvinyl siloxane with a double impression 2-step puttywash technique, while the antagonist teeth (lower jaw) were printed using irreversible hydrocolloid (Figure 8).
The next stage is making a bite registration using polyvinyl siloxane. The Vaseline application was then followed by the insertion of a temporary inlay (bis-acryl composite) (Figure 9).
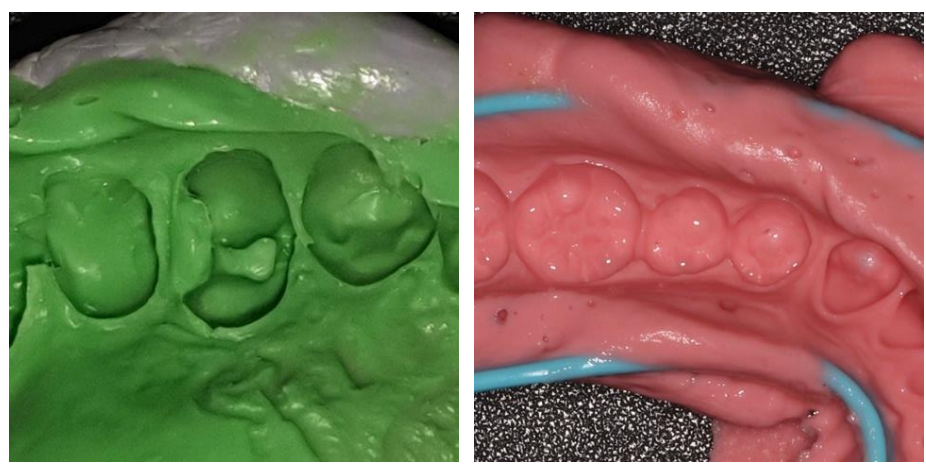

Figure 8. Impression of maxillary (left) and antagonistic jaws (right) working models.
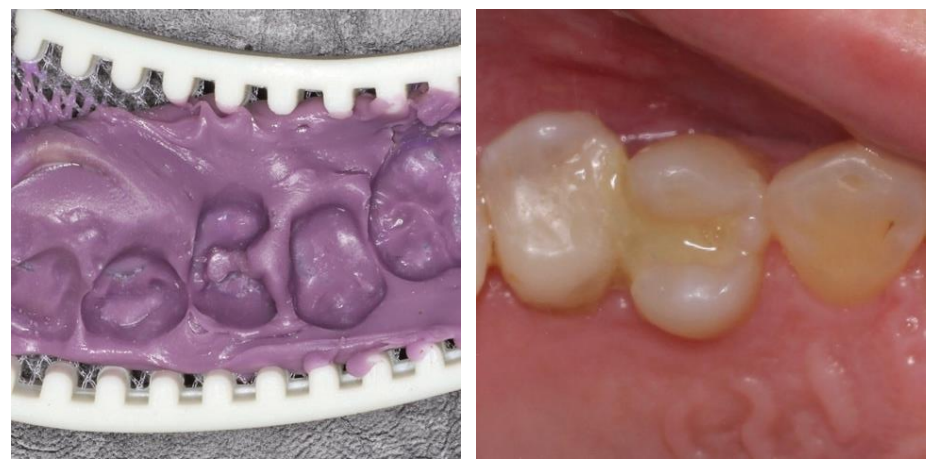

Figure 9. Result of bite registration (left) and the result of temporary inlay (right).
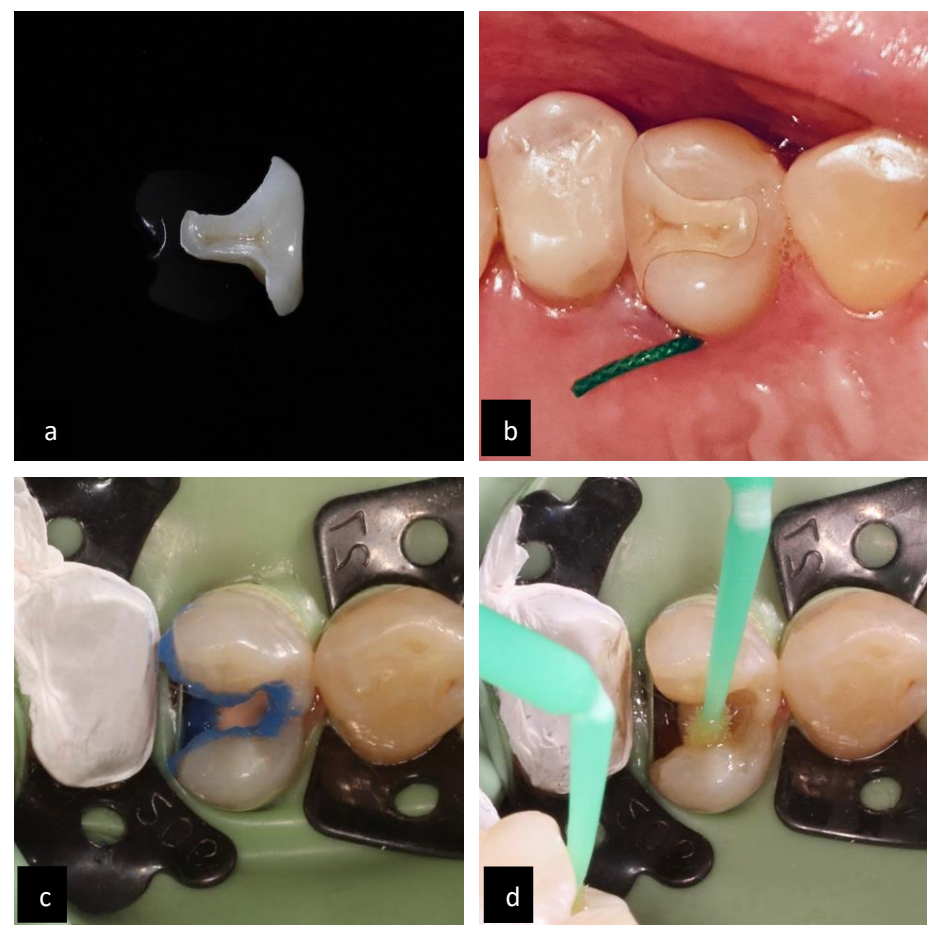

Figure 10.a) Zirconia inlays; b) Try in inlays; c) Selective etch gear 24; d) Universal adhesive. 
On the third visit (9-8-2021), the temporary inlay was removed for trial and error of the zirconia inlay and adjustment of occlusion, proximal contact, anatomy, and colour. The working area was then isolated, and the tooth surface was decontaminated using a rotary brush and pumice. The next step is etching and adhesive, then applying silane 10-MDP universal primer on the inner surface inlay for 60 seconds and applying a light breeze for 5 seconds (Figure 10).

Zirconia inlay cementation shows in Figure 11 using selective etch adhesive resin cement dual-cure, initial curing for 2 seconds. The remaining cement is cleaned, followed by final light curing for 20 seconds from all directions. After that, finishing and polishing were carried out using Eve Diacera, then checking for occlusion, an adaptation of margins, proximal contacts, and x-rays

At the third visit, the patient was instructed to come for control ten days later (19-8-2021). The patient stated no complaints, extraoral abnormalities were found, and on intraoral examination, the percussion test was negative (-), the zirconia inlay was in good condition, and the surrounding gingiva was normal (Figure 12).

\section{DISCUSSION}

Clinical zirconia has been known as a gem since ancient times. The name zirconium comes from the Arabic "zargun" (golden colour), which in turn comes from the two Persian words "zar" (gold) and "gun" (colour). Zirconia is a crystalline dioxide of zirconium. Zirconium oxide was first used for media purposes in 1969 for orthopedic applications. Zirconia was later proposed as a new material for a head hip replacement for titanium or alumina prostheses. Patients and dentists have been searching for tooth-colored, metal-free restorative materials as interest in aesthetics and concerns about toxic and allergic reactions to certain materials have increased. Therefore, the recent development of highstrength dental ceramics, which are less brittle, have good tensile strength, and are less affected by stress resulting in restoration failure, are of great interest to prosthetic dentistry, where strength and esthetics are critical. ${ }^{7}$

Zirconia is divided into three distinct patterns: monoclinic (M), tetragonal (T), and cubic (C). Pure zirconia is monoclinic at room temperature and remains stable up to $1170^{\circ} \mathrm{C}$. Above this temperature, it changes to tetragonal and
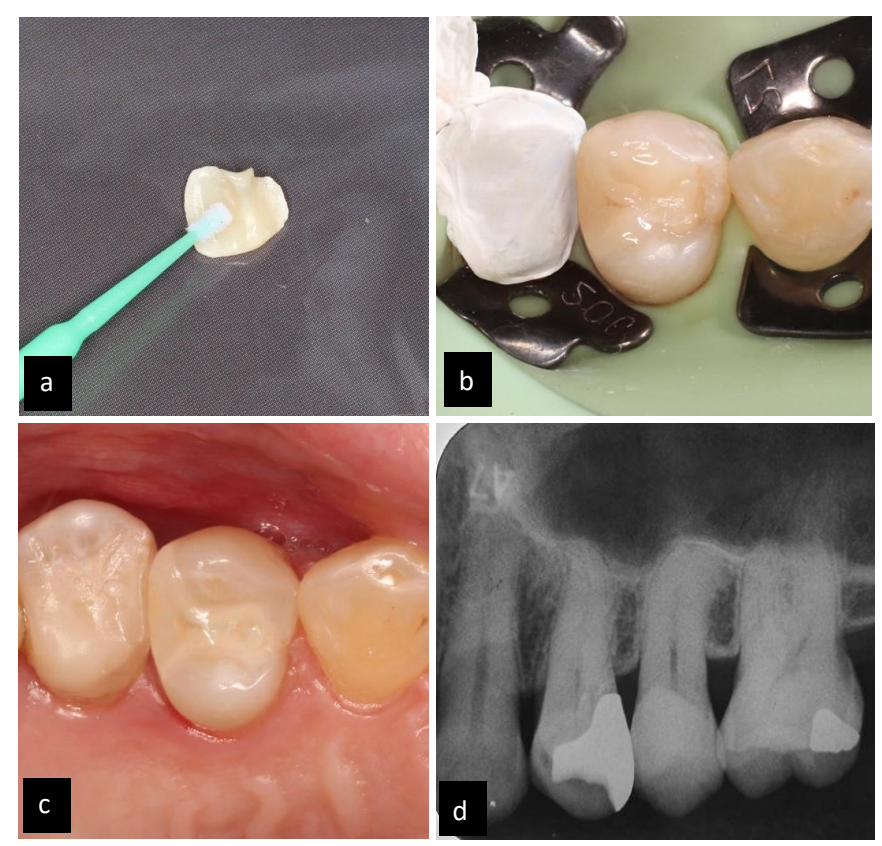

Figure 11. a) Primary universal 10-MDP silane application; b) Insertion of inlays; c) Finishing and polishing; d) X-rays.

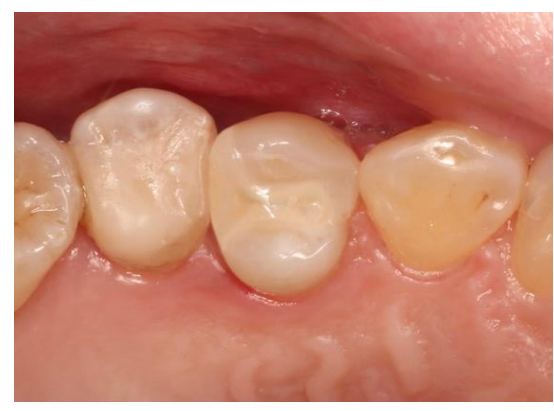

Figure 12. Clinical photos after treatment. 
then to the cubic phase, which exists up to the melting point at $2370^{\circ} \mathrm{C}$. During cooling, the tetragonal phase changes back to monoclinic in temperatures ranging from $100^{\circ} \mathrm{C}$ to $1070^{\circ} \mathrm{C}$. Many types of ceramics containing zirconia are currently available, but only three are used in dentistry. The types are cation-doped tetragonal yttrium zirconia polycrystals (3Y-TZP), partially stabilized magnesium cation-doped zirconia (Mg-PSZ) and alumina hardened zirconia (ZTA). In dentistry itself, zirconia is often used as posts, jacket crowns and bridges, implant abutments and inlays and onlays. ${ }^{7}$

From an aesthetic point of view, Zirconia has a dull white colour, and its opacity can cover the underlying structure. Most of the zirconia systems used in dental restorations have added structural stains to improve esthetics. ${ }^{8}$ Recently, zirconia materials have also been developed with translucency repair staining to match the colour of human teeth. ${ }^{6}$

The mechanical strength of zirconia as a dental material is classified as having the highest strength and good fracture toughness. ${ }^{9}$ Zirconia material has a much better hardness than composite resin materials and can even cause long-term abrasion of the opposing teeth. ${ }^{10}$ Posterior tooth restorations have at least compression strength. Of $1000 \mathrm{~N}$ to ensure long-term restoration success and to allow material fatigue. Given the maximum force of $400 \mathrm{~N}$ in the anterior area and $600 \mathrm{~N}$ in the posterior oral area, only zirconia can guarantee the initial strength required for dental restoration. Zirconia material withstands many times the level of load that occurs in the mouth after treatment. ${ }^{9}$

\section{CONCLUSION}

In recent years, many all-ceramic materials have been introduced in dentistry. One of which is zirconia, which is becoming popular in dentistry due to its good mechanical properties and biocompatibility. Recently, zirconia has also been developed staining with improved translucency so that it becomes more aesthetic. Zirconia has a higher level of material resistance than other restorative materials such as composites. This is what makes zirconia the choice, especially for use as a framework for all-ceramic and partially-fixed crowns dental prosthesis.

\section{REFERENCES}

1. Kumala P, et al,. Kamus Saku Kedokteran Dorland. Jakarta: EGC. 2006.

2. Widayati N. Factors Associated with Dental Caries in Children Aged 4-6 Years Old. Jurnal Berkala Epidemiologi. 2014; 2(2): 196-205.

3. Nurhidayati O, Eram TP \& Bambang W. Perbandingan Media Power Point dengan Flip Chart dalam Meningkatkan Pengetahuan Kesehatan Gigi dan Mulut. Unnes Journal of Public Health. 2012; 1(1): 31-35.

4. Hanen B, Mariem B, Hayet H, Nouha M \& Lassaad K. Clinical Follow Up of Aesthetic Inlays and Onlays: Systematic Literature Review. WJPMR. 2018; 4(12): 112-121.

5. Aspros A. Inlays \& onlays clinical experiences and literature review. J Dent Health Oral Disord Ther. 2015; 2(1): 26-31.

6. Nistor L, Gradinaru M, Rica R, Marasescu P, Stan M, Manolea H, Augustin I \& Moraru I. Zirconia Use in Dentistry-Manufacturing and Properties. Curr Health Sci J. 2019; 45(1): 28-35.

7. Madfa A, Al-Sanabani F, Al-Qudami N, Al-Sanabani J \& Amran A. Use of Zirconia in Dentistry: An Overview. The Open Biomaterials Journal. 2014; 5: 1-9.

8. Bona AD, Oscar EP \& Alessandretti, R. Zirconia as a Dental Biomaterial. Materials. 2015; 8: 4978-4991.

9. Daou E \& Al-Gotmeh M. Zirconia Ceramic: A Versatile Restorative Material. Dentistry. 2014; 4: 219.

10. Dhoum S, Jabrane K, Dhaimy S, Talache N, Lahlou K, Ouazzani A \& Merini H. Indirect Posterior Restoration: Composite Inlays. Biomed J Sci \&Tech Res. 2018; 5(1): 4312-4316. 